\title{
Journal of Cellular Physiology
}

WILEY

\section{Virtual screening identifies a PIN1 inhibitor with possible anti-ovarian cancer effects}

\begin{tabular}{|r|l|}
\hline Journal: & Journal of Cellular Physiology \\
\hline Manuscript ID & Draft \\
\hline Diley - Manuscript type: & Original Research Article \\
\hline Author: & n/a \\
\hline Complete List of Authors: & $\begin{array}{l}\text { Russo Spena, Concetta; Centro Riferimento Oncologico, Translational } \\
\text { medicine } \\
\text { De Stefano, Lucia; Centro di Riferimento Oncologico } \\
\text { Poli, Giulio; Universita degli Studi di Pisa Dipartimento di Farmacia } \\
\text { Granchi, Carlotta; Universita degli Studi di Pisa Dipartimento di Farmacia } \\
\text { El Boustani, Maguie; Centro di Riferimento Oncologico } \\
\text { Ecca, Fabrizio; Centro di Riferimento Oncologico } \\
\text { Grassi, Gabriele; University of Trieste } \\
\text { Grassi, Mario; Universita degli Studi di Trieste Dipartimento di } \\
\text { Ingegneria e Architettura } \\
\text { Canzonieri, Vincenzo; Centro di Riferimento Oncologico, IRCCS, } \\
\text { Anatomia Patologica } \\
\text { Giordano, Antonio; Temple University, Biology } \\
\text { Tuccinardi, Tiziano; University of Pisa } \\
\text { Caligiuri, Isabella; National Cancer Institute and Center for Molecular } \\
\text { Biomedicine, Division of Experimental and Clinical Pharmacology, } \\
\text { Department of Molecular Biology and Translational Research } \\
\text { Rizzolio, Flavio; Universita Ca' Foscari, DSMN }\end{array}$ \\
\hline Key Words: & \begin{tabular}{l} 
Pin1, ovarian cancer, small molecule inhibitors, consensus docking \\
\hline
\end{tabular} \\
\hline
\end{tabular}

\section{SCHOLARONE Manuscripts}




\section{Virtual screening identifies a PIN1 inhibitor with possible anti-ovarian cancer effects}

Running title: PIN1 inhibitor with anti-cancer effects

Concetta Russo Spena ${ }^{1,2^{*}}$, Lucia De Stefano ${ }^{1,2^{*}}$, Giulio Poli ${ }^{3}$, Carlotta Granchi ${ }^{3}$, Maguie El Boustani ${ }^{1,4}$, Fabrizio Ecca ${ }^{5}$, Gabriele Grassi ${ }^{6}$, Mario Grassi ${ }^{7}$, Vincenzo Canzonieri ${ }^{1}$, Antonio Giordano ${ }^{8}$, Tiziano Tuccinardi $^{3,8}$, Isabella Caligiuri ${ }^{1 \S}$ and Flavio Rizzolio ${ }^{1,8,9 \S}$

1 Pathology Unit, National Cancer Institute, Aviano, Italy.

2 Doctoral School in Chemistry, University of Trieste, Italy.

3 Department of Pharmacy, University of Pisa, Italy.

4 Doctoral School in Molecular Biomedicine, University of Trieste, Italy.

5 Experimental and Clinical Pharmacology, National Cancer Institute, Aviano, Italy

6 Department of Life Sciences, Cattinara University Hospital, University of Trieste, Italy.

7 Department of Engineering and Architecture, University of Trieste, Italy.

8 Sbarro Institute for Cancer Research and Molecular Medicine, Center for Biotechnology, College of Science and Technology, Temple University, Philadelphia, Pennsylvania.

9 Department of Molecular Sciences and Nanosystems, Ca' Foscari University, Venezia-Mestre, Italy.

Keywords: Pin1, ovarian cancer, small molecule inhibitors, consensus docking

${ }^{*}$ Co-first authors

$\S$ Corresponding authors

\section{Flavio Rizzolio}

Department of Molecular Sciences and Nanosystems, Ca' Foscari University, Venezia-Mestre, Italy. Tel. +39-0434 659026

Fax. $+39-0434659370$

E-mail: flavio.rizzolio@unive.it 


\author{
Isabella Caligiuri \\ Pathology Unit, National Cancer Institute, Aviano, Italy \\ Tel. +39-0434 659026 \\ Fax. $+39-0434659370$ \\ E-mail: icaligiuri@cro.it
}

\title{
Acknowledgments
}

The authors are grateful to the Associazione Italiana per la Ricerca sul Cancro - My First AIRC grant (n. 1569 to FR). Valerie Matarese provided scientific editing. 


\footnotetext{
1 ABSTRACT

2 PIN1 is a peptidyl-prolyl isomerase that binds phospho-Ser/Thr-Pro motifs in proteins and catalyzes the

3 cis-trans isomerization of proline peptide bonds. PIN1 is overexpressed in several cancers including

4 high-grade serous ovarian cancer. Since few therapies are effective against this cancer, PIN1 could be a

5 therapeutic target but effective PIN1 inhibitors are lacking.

6 To identify molecules with in vivo inhibitory effects on PIN1, we used consensus docking to model

7 existing PIN1-ligand X-ray structures and to screen a chemical database for candidate inhibitors. Ten

8 molecules were selected and tested in cellular assays, leading to the identification of VS10 that bound and

9 inhibited PIN1. VS10 treatment reduced viability of ovarian cancer cell lines by inducing proteasomal

10 PIN1 degradation, without effects on PIN1 transcription, and also reduced levels of downstream targets $\beta$ -

11 catenin, cyclin D1 and pSer473-Akt.

12 VS10 is a selective PIN1 inhibitor that may offer new opportunities for treating PIN1-overexpressing 13 tumors.

14

15
} 


\section{INTRODUCTION}

17 In cancer, molecularly targeted therapy is attractive since it may not have the adverse effects commonly

18 associated with chemotherapy. On the other hand, blocking only one molecular pathway may be

19 ineffective since cancer cells have many alternative routes for staying alive and multiplying, allowing the

20 neoplasm to progress lethally. The inhibition of proteins that control multiple oncogenic pathways could

21 be the solution (Ciarcia et al., 2013).

22 One protein that regulates multiple cellular pathways is PIN1 (peptidylprolyl cis/trans isomerase, NIMA-

23 interacting 1). This enzyme catalyzes the cis-trans conformational switch of the proline peptide bond in

24 Ser/Thr-Pro motifs, which are target sequences of kinases and phosphatases (Lu et al., 1996).

25 Phosphorylation and dephosphorylation of Ser/Thr-Pro motifs is a common signaling mechanism in cell

26 growth and transformation, and the activities of different kinases and phosphatases depend on the

27 conformational state of the proline peptide bond in their target motifs ( $\mathrm{Lu}$ et al., 2007). Hence,

28 isomerization of Ser/Thr-Pro motifs affects phosphorylation status of many proteins, with profound

29 effects on their functions and stability (Lucchetti et al., 2013; La Montagna et al., 2012, 2013, Rizzolio et

30 al., 2012, 2013; Russo Spena et al., 2018).

31 Isomerization and phosphorylation of Ser/Thr-Pro motif-containing proteins is implicated in the 32 activation of oncogenes and inactivation of tumor suppressor genes ( $\mathrm{Lu}$ et al., 2007). Indeed, in multiple 33 tumors, PIN1 expression was found to be upregulated and to correlate with poor prognosis (Bao et al., 34 2004). In mice, Pin1 overexpression induced chromosome instability and tumorigenesis (Suizu et al., 35 2006), while Pin1 knockout was not lethal but caused various cell-proliferative alterations, such as 36 testicular and retinal atrophy and lower body weight (Liou et al., 2002). Moreover, in Pin1 knockout 37 female pregnant mice the mammary gland has a severe deficiency in the development and proliferation of 38 mammary epithelial cells (Liou et al., 2002). We recently discovered that PIN1 is overexpressed in human 39 serous ovarian cancer, and showed that its inhibition induces tumor cell death and tumor shrinkage in a 
40 immunocompetent mouse model of metastatic ovarian cancer (Russo Spena et al., 2018). These

41 observations suggest that PIN1 is a strong candidate for targeted therapy.

42 Over the past 10 years, several PIN1 inhibitors have been developed and shown to have high potency in 43 biochemical assays (Moore and Potter, 2013). However, many of these molecules have limited activity in 44 cells due to the presence of the doubly negative-charged phosphate group, which mimics natural phospho45 substrates but limits their cell permeability (Guo et al., 2009). Non-phosphate inhibitors with low- to sub46 micromolar activity in cells have also been described (Campaner et al., 2017; Subedi et al., 2016; Zhao et 47 al., 2016). Among these, all-trans retinoic acid (ATRA) has been extensively studied. ATRA is approved 48 for the treatment of acute promyelocytic leukemia (APL), which is almost caused by aberrant 49 promyelocytic leukemia-retinoic acid receptor $\alpha$ (PML-RAR $\alpha)$ (Johnson and Redner, 2015). As reported 50 by Wei et al. (Wei et al., 2015), PIN1 binds PML-RAR $\alpha$ and stabilizes it, but ATRA inhibits PIN1 and 51 leads to its degradation, thereby destabilizing PML-RAR $\alpha$ and inhibiting the growth of APL cells. These 52 same researchers tested ATRA on triple negative breast cancer cells and found that it inhibited their 53 growth via PIN1 inhibition. Yet ATRA has a short half-life (Regazzi et al., 1997) and is not specific for 54 PIN1 (Notario et al., 2003; Ochoa et al., 2003; Schenk et al., 2014), limiting its use.

55 One approach for discovering new PIN1 inhibitors is virtual screening using a consensus docking 56 protocol. Docking is an in silico modeling technique that predicts the most energetically favored position 57 of a ligand bound to a protein, while consensus docking combines the results of different docking 58 methods to obtain better results from both a qualitative and a quantitative point of view (Poli et al., 2016; 59 Tuccinardi et al., 2014b). We found that the consensus approach was better than single-docking methods 60 in predicting ligand binding poses, and that as the consensus level (i.e. the number of docking methods 61 yielding the same pose) of a docking pose increased, so did its reliability. The reliability of this approach 62 was shown in virtual screening campaigns that identified new non-covalent inhibitors for three different 63 enzymes (Chiarelli et al., 2018; Poli et al., 2015; Tuccinardi et al., 2014a). 
64 Encouraged by the results obtained with both the in silico and experimental tests, we applied consensus 65 docking to the identification of new PIN1 inhibitors. We found a new compound, VS10, with micromolar 66 efficacy and possible anticancer effects. Treatment of ovarian cancer cells with VS10 led to PIN1 67 degradation by the proteasome and reduced the cellular levels of the PIN1 downstream targets $\beta$-catenin, 68 cyclin D1 and pSer473-Akt. These data suggest that VS10 is a potential new therapeutic agent in PIN169 overexpressing tumors. 
$\begin{array}{lll}70 & 2 & \text { Materials and Methods }\end{array}$

$71 \quad 2.1$ Molecular modeling

72 The 12 available human PIN1-ligand X-ray complexes were retrieved from the Protein Data Bank

73 (Berman et al., 2000). For all complexes, the ligand was extracted from its X-ray structure and subjected

74 to a conformational search. To test the reliability of consensus docking in predicting the position of the

75 ligand binding site, each ligand was docked in all the PIN1 3D structures using ten docking procedures,

76 namely AutoDock 4.2.3, DOCK 6.7, FRED 3.0, Glide 5.0 (SP and XP), GOLD 5.1 (ASP, ChemScore,

77 GoldScore and PLP), and AutoDock Vina 1.1, as previously described (Poli et al., 2018; Tuccinardi et al.,

78 2015). The reliability of these docking procedures was evaluated in cross-docking analyses. For each

79 procedure, we calculated the average root-mean-square deviation (RMSD) between the position of the

80 ligand predicted by the docking and the known, experimental position, for all the ligands docked into all

81 of the binding sites. The procedure with the lowest average RMSD was considered the most reliable.

82 Details about the docking procedures and the cross-docking analyses are given in Supplementary

83 Materials and Methods.

84 To study the effects of consensus docking on the docking evaluations, for each ligand docked into each

85 PIN1 binding site, we clustered the results of the ten docking procedures, to search for common binding

86 modes. For this purpose, consensus level was defined as the number of docking poses that clustered

87 together. At each consensus level, we calculated average RMSD and the percentage of compounds 88 retained ("survived").

89 To screen for new PIN1 inhibitors using consensus, a hierarchical workflow was used to apply the ten 90 docking procedures to a subset of the Enamine database (HTS Collection) comprising the approximately 9132,500 compounds with at least one negative charge. Compounds with a consensus level of ten were 92 selected. To verify the stability of their binding mode as predicted by docking calculations, we did $10 \mathrm{~ns}$ 93 molecular dynamic simulations with explicit water (Supplementary Materials and Methods). We 94 calculated the average RMSD of the position of each ligand during the simulation compared to their 
95 initial docking pose, and analyzed the stability of the interactions predicted by docking. Compounds with

96 an average RMSD $<2.0 \AA$ were selected and purchased from Enamine (Monmouth Junction, NJ, USA)

97 for study in cellular assays.

98

$99 \quad 2.2$ Cell lines and reagents

100 Human OVCAR3 and SKOV3 cell lines were purchased from ATCC (Manassas, VA, USA). Human

101 OVCAR5 and NIH3T3 cell lines were provided by Gustavo Baldassarre (Aviano, Italy, EU) while the 102 A2780 human ovarian cancer cell line was provided by Donatella Aldinucci (Aviano, Italy, EU). Cell 103 lines were grown in RPMI-1640 medium with 10\% fetal bovine serum. All the cell lines tested negative 104 for mycoplasma contamination by PCR analysis and gel electrophoresis.

105 Antibodies used in western blotting included: mouse anti-HSP70 (1:1000; cat. no. sc 24) and rabbit anti106 PIN1 (1:250; cat. no. sc-15340) from Santa Cruz Biotechnology (Santa Cruz, CA USA); mouse anti107 human cyclin D1 (1:1000; cat. no. 556470) from BD Pharmingen (Franklin Lakes, NJ, USA); rabbit anti$108 \beta$-catenin (1:1000; cat. no. 8480S), rabbit anti-pSer473-Akt 1:1000; cat. no. 4060s) and rabbit anti- $\beta$-actin 109 (1:1000; cat. no. 4967S) from Cell Signaling Technology (Danvers, CO, USA). Secondary antibodies 110 were mouse anti-rabbit IgG (1:5000; cat. no. 31464) and goat anti-mouse IgG (1:5000; cat. no. 31432) 111 from Thermo Fisher Scientific (Waltham, MA, USA).

1132.3 Half-maximal inhibitory concentration $\left(\mathrm{IC}_{50}\right)$

114 Compounds identified by virtual screening were tested for PIN1 inhibitory activity using the in vitro 115 fluorescent SensoLyte Green Pin1 Assay Kit (AS-72240; AnaSpec, Fremont, CA, USA). Compounds 116 were serially diluted 1:10 starting from $1 \mathrm{mM}$. ATRA was used as positive control.

$117 \mathrm{IC}_{50}$ was also calculated from cell viability. Briefly, cells were plated in 96-well plates at $5 \times 10^{2}$ 118 cells/well. The next day, cells were treated with VS10 in 1:2 serial dilutions from $300 \mu \mathrm{M}$ to $2.3 \mu \mathrm{M}$. 
119 After $96 \mathrm{~h}$, cell viability was evaluated using the CellTiter-Glo Luminescent Cell Viability Assay 120 (Promega, Fitchburg, WI, USA) with the Infinite M1000 PRO microplate reader (Tecan, Mannedorf, 121 Switzerland). $\mathrm{IC}_{50}$ was calculated using Prism software (GraphPad, La Jolla, CA, USA).

122

$123 \quad 2.4$ Cell treatments

124 To test effects on PIN1 protein stability, NIH3T3 cells were plated in 100 X $20 \mathrm{~mm}$ tissue culture dishes $125\left(1.5 \times 10^{5}\right.$ cells per dish). One day later, cells were treated with 0,35 and $70 \mu \mathrm{M}$ compound VS10 for 48 $126 \mathrm{~h}$, then with $10 \mu \mathrm{M}$ MG132 or vehicle (DMSO) for $6 \mathrm{~h}$ (Roberti et al., 2011). In other experiments, cells 127 were treated with $70 \mu \mathrm{M}$ VS10 or vehicle (DMSO) for $24 \mathrm{~h}$, followed by $10 \mu \mathrm{g} / \mathrm{mL}$ cycloheximide for 0 , $1283,6,12$ and $24 \mathrm{~h}$. After treatments, cells were collected for western blotting and RT-PCR.

129 To test effects on PIN1 targets, OVCAR3 cells were seeded in 100 X $20 \mathrm{~mm}$ tissue culture dishes $(5 \mathrm{X}$ $13010^{5}$ cells per dish). One day later, cells were treated with $70 \mu \mathrm{M}$ VS10 or $10 \mu \mathrm{M}$ ATRA. After treatment, 131 cells were collected for western blotting.

$133 \quad 2.5$ Western blotting

134 A total cell extract was obtained by lysing cells with RIPA buffer plus protease and phosphatase 135 inhibitors (Complete-EDTA-free Protease Inhibitor Cocktail; Roche, Basel, Switzerland), incubating on 136 ice for $20 \mathrm{~min}$, sonicating for $5 \mathrm{~s}$, and centrifuging at $12,000 \mathrm{rpm}$ for $20 \mathrm{~min}$ at $4{ }^{\circ} \mathrm{C}$. Equal amounts of 137 protein $(30 \mu \mathrm{g})$ were separated on TruPAGE Precat Gels 4-12\% (Sigma-Aldrich, St. Louis, MI, USA). 138 Proteins were transferred onto nitrocellulose membranes (Amersham Protran $0.45 \mu \mathrm{m}$; GE Healthcare 139 Life Sciences, Chicago, IL, USA). Free protein-binding sites were blocked for 30 min with 5\% non-fat 140 dried milk in TBS containing $0.1 \%$ Tween-20 (TBS-T). The membranes were incubated with primary 141 antibodies against PIN1 and $\beta$-actin at $4{ }^{\circ} \mathrm{C}$ overnight, washed three times with TBS-T, and incubated 142 with an HRP-conjugated secondary antibody for $1 \mathrm{~h}$ at room temperature. Bound antibodies were detected 
143 using LiteAblot PLUS Enhanced Chemiluminescent Substrate (EuroClone Life Sciences, Pero, Italy, 144 EU). The results were analyzed with the ChemiDoc imaging system (Bio-Rad, Hercules, CA, USA).

145

$146 \quad 2.5$ RNA extraction and PCR

147 Total RNA was prepared from murine NIH3T3 cells using the Smarter Nucleic Acid Sample Preparation 148 kit (Stratec Molecular; Berlin, Germany, EU). Total RNA (400 ng) was reverse transcribed in a $10 \mu \mathrm{l}$ 149 reaction using GoScript Reverse Transcription System kit (Promega, Fitchburg, WI, USA). cDNA (0.1 150 volume) was amplified using GoTaq G2 Polymerase and Master Mix (Promega, Fitchburg, WI, USA). 151 Murine Hprt was used as a control. Amplification reactions were carried out in a final volume of $20 \mu 1$ as 152 follows: $5 \mathrm{~min}$ at $95{ }^{\circ} \mathrm{C} ; 20 \mathrm{~s}$ at $95{ }^{\circ} \mathrm{C}, 30 \mathrm{~s}$ at $60{ }^{\circ} \mathrm{C}, 30 \mathrm{~s}$ at $72{ }^{\circ} \mathrm{C} \times 30$ cycles. The products were 153 analyzed via 3\% agarose gel electrophoresis. 


\section{RESULTS}

155 3.1 Virtual screening prioritizes ten new PIN1 inhibitors

156 As a first step to screening for new PIN1 inhibitors, we tested the reliability of consensus docking in 157 predicting the position of the ligand binding site for existing PIN1-ligand X-ray complexes. Ligands were 158 extracted from their X-ray complexes and then docked in all the structures using 10 docking procedures. 159 A total of 12 ligand-protein structures were analyzed, with 1440 docking calculations. Reliability was 160 assessed from the average root-mean-square deviation (RMSD) between the position of the ligand 161 predicted by docking and their known position. As shown in Figure 1, the docking procedures had an 162 average RMSD in the range of 3.7-4.9 $\AA$, with AutoDock4 having the best result (smallest deviation).

163 Then, the results of each docking procedure (data for each ligand docked into each PIN1 binding site) 164 were clustered to search for common binding modes. As the consensus level increased from 2 (i.e., taking 165 into account all the ligand-protein combinations that showed at least two out of ten docking poses 166 clustered together) to the maximum value of 10 , the average RMSD decreased from $3.7 \AA$ to $0.7 \AA$ 167 (Figure 2). The best reliability achieved with consensus docking (0.7 $\AA$ at consensus 10$)$ is about 5-times 168 better than that obtained by using the best docking procedure in the cross-docking analysis $(3.7 \AA$ with 169 AutoDock). However, as the consensus level increased, the percentage of all ligand-protein combinations 170 retained ("survived") decreased, from $99 \%$ at a consensus level of 2 to $5 \%$ at a consensus of 10 . These 171 results mean that the quality of docking predictions increases with the consensus level, and that consensus 172 docking improves the prediction of the ligand docking pose.

173 Consensus docking was then used in virtual screening for new PIN1 inhibitors. The 10 docking 174 procedures were applied to a filtered Enamine database, and 32 compounds (out of about 32,500 175 compounds screened) reached a consensus of 10 . These 32 compounds were subjected to molecular 176 dynamic simulations, to examine the stability of their binding. A total of 10 compounds had an average 177 RMSD (between the position of the 32 ligands during the simulation and their initial docking poses) $<2.0$ 
$178 \AA$. These compounds were obtained for testing in biological assays to evaluate their PIN1 inhibitory 179 activity.

180

1813.2 Compound VS10 is a potent PIN1 inhibitor

182 Ten compounds selected by virtual screening were tested for inhibitory action against PIN1 isomerization 183 in a fluorescent assay with a logarithmic dilution from $1 \mathrm{mM}$ to $1 \mathrm{nM}$. The half-maximal inhibitory 184 concentration $\left(\mathrm{IC}_{50}\right)$ was $>100 \mu \mathrm{M}$ for nine compounds (Table 1). One compound (called VS10) had a 185 mean $\mathrm{IC}_{50}=13.4 \mu \mathrm{M}(\mathrm{SD}=1.24 \mu \mathrm{M})$. The positive control, ATRA, showed an $\mathrm{IC}_{50}$ of $33.2 \mu \mathrm{M}$ (in the 186 range of published results (Liao et al., 2017)), which is about 2-fold less than that of VS10.

187 Figure 3 shows the binding of VS10 in the PIN1 binding site. The carboxylic group of the ligand has 188 ionic interactions with $\mathrm{R} 69$ and $\mathrm{K} 63$, the thiophene ring interacts with $\mathrm{C} 113$, and the phenyl ring is 189 inserted into a lipophilic cleft mainly delimited by L122, M130, F134 and H157. The 3190 methylbenzofuran-2-carboxamide fragment makes an H-bond with the hydroxyl oxygen of S154 and is 191 partially exposed to water.

192

193 3.3 Compound VS10 reduces cancer cell viability

194 The activity of VS10 was tested in human OVCAR3 and OVCAR5 cell lines, as models of high-grade 195 serous ovarian cancer, and in human SKOV3 and A2780 ovarian cancer cell lines. Cells were exposed to 196 serial dilutions of the drug for $96 \mathrm{~h}$, and $\mathrm{IC}_{50}$ values were calculated from cell viability. VS10 showed $\mathrm{IC}_{50}$ 197 values ranging from 53.9 to $76.4 \mu \mathrm{M}$ (Table 2).

199 3.4 Compound VS10 induces PIN1 protein degradation

200 Knowing that high affinity or covalent PIN1 inhibitors induce the protein's degradation, we examined the 201 effects of VS10 on PIN1 levels in NIH3T3 fibroblasts. Treatment of NIH3T3 cells with two 
202 concentrations of VS10 seemed to reduce the steady-state amount of this protein, and this effect was

203 blocked by MG132 proteasomal inhibitor (Figure 4a). When cells were first treated with VS10 and then

204 with cycloheximide (to inhibit protein synthesis), the level of PIN1 protein decreased over time (Figure

2054 b, right panel); this effect was not seen when cells were treated with DMSO (vehicle) and cycloheximide

206 (Figure 4b, left panel). VS10 had no effect on the level of PIN1 mRNA (Figure 4c).

207 Finally, the effects of PIN1 inhibition on three PIN1 targets were assessed in the OVCAR3 cell line.

208 Western blotting showed that VS10 treatment decreased the levels of $\beta$-catenin, cyclin D1 and pSer473-

209 Akt proteins (Figure 5). 


\section{Conclusions}

211 In this study, we used consensus docking following by molecular dynamic simulations to identify

212 possible PIN1 inhibitors. By applying this procedure to a dataset of ligands, we selected 10 potential

213 PIN1 inhibitors for analysis. An in vitro assay for inhibitory activity against PIN1 isomerization revealed

214 that VS10 had an $\mathrm{IC}_{50}$ in the low micromolar range $(13.4 \pm 1.2 \mu \mathrm{M})$. This molecule was selected for 215 further study and was found to have inhibitory effects on four ovarian cancer cell lines, with $\mathrm{IC}_{50}$ values 216 ranging from 53.9 to $76.4 \mu \mathrm{M}$.

217 Structurally, VS10 includes a 3-methylbenzofuran-2-carboxamide fragment, a thiophene and a phenyl 218 ring. Molecular modeling showed that each VS10 moiety interacts with distinct residues in the PIN1 219 catalytic site: the VS10 carboxylic group forms ionic bonds with PIN1 R69 and K63; the thiophenic ring 220 interacts with $\mathrm{C} 113$; the phenyl ring is inserted into a lipophilic cleft mainly delimited by L122, M130, 221 F134 and H157; and the 3-methylbenzofuran-2-carboxamide fragment forms an H-bond with the 222 hydroxyl oxygen of S154.

223 VS10 treatment of NIH3T3 cells induced PIN1 degradation via the proteasome, as shown by the fact that 224 treatment with MG132 (a proteasome inhibitor) restored the normal level of PIN1 in VS10-treated cells. 225 These results are similar to those obtained with another PIN1 inhibitor, ATRA (Wei et al., 2015). The 226 inhibitory effects of VS10 on PIN1 seen here did not involve a change in PIN1 transcription. As a 227 consequence of decreased PIN1 expression, the levels of the PIN1 downstream targets $\beta$-catenin, cyclin 228 D1 and pSer473-Akt also decreased.

229 Several studies reported an intriguing correlation between the expression levels of PIN1 and its 230 downstream targets in maintaining the survival and proliferation of cancer cells, with PIN1 regulating 231 cyclin D1 expression both directly and indirectly as a consequence of its interactions with $\beta$-catenin and 232 pSer473-Akt (Liao et al., 2009; Liou et al., 2002; Ryo et al., 2001). In particular, PIN1 prevents $\beta$-catenin 233 degradation by adenomatous polyposis coli (APC) (Ryo et al., 2001). On the other hand, $\beta$-catenin 234 stabilization and activation may result from PIN1-mediated activation of Akt (Liao et al., 2009). In 
235 accordance, we found that $\beta$-catenin, cyclin D1 and pSer473-Akt were deregulated in VS10-treated cells.

236 The simultaneous alteration of different pathways regulated by PIN1 and involved in cancer progression

237 suggests that VS10 is a candidate drug.

238 In conclusion, VS10 has the potential to be a more efficient PIN1 inhibitor than existing molecules, with 239 possible clinical application in PIN1-overexpressing cancers such as high-grade serous ovarian cancer.

240 Further studies are required to test the efficacy and safety of this molecule.

241

242 Competing interests

243 The authors declare no competing interests.

244 Acknowledgment

245 FR and IC are thankful to Associazione Italiana per la Ricerca sul Cancro (AIRC). MyFirst AIRC, $246 \quad \mathrm{n}^{\circ} 15639$

247 Supporting information

248 Additional supporting information may be found in the online version of this article at the publisher's 249 website.

250 


\section{$251 \quad$ References}

252 Bao L, Kimzey A, Sauter G, Sowadski JM, Lu KP, Wang DG. 2004. Prevalent overexpression of prolyl 253 isomerase Pin1 in human cancers. Am J Pathol 164(5):1727-1737.

254 Berman HM, Westbrook J, Feng Z, Gilliland G, Bhat TN, Weissig H, Shindyalov IN, Bourne PE. 2000. 255 The Protein Data Bank. Nucleic Acids Res 28(1):235-42.

256 Campaner E, Rustighi A, Zannini A, Cristiani A, Piazza S, Ciani Y, Kalid O, Golan G, Baloglu E, 257 Shacham S, Valsasina B, Cucchi U, Pippione AC, Lolli ML, Giabbai B, Storici P, Carloni P, Rossetti G, 258 Benvenuti F, Bello E, D’Incalci M, Cappuzzello E, Rosato A, Sal G Del. 2017. A covalent PIN1 inhibitor 259 selectively targets cancer cells by a dual mechanism of action. Nat Commun 8:15772.

260 Chiarelli LR, Mori M, Barlocco D, Beretta G, Gelain A, Pini E, Porcino M, Mori G, Stelitano G, 261 Costantino L, Lapillo M, Bonanni D, Poli G, Tuccinardi T, Villa S, Meneghetti F. 2018. Discovery and 262 development of novel salicylate synthase (MbtI) furanic inhibitors as antitubercular agents. Eur J Med 263 Chem 155:754-763.

264 Ciarcia R, Damiano S, Montagnaro S, Pagnini U, Ruocco A, Caparrotti G, D’Angelo D, Boffo S, Morales 265 F, Rizzolio F, Florio S, Giordano A. 2013. Combined effects of PI3K and SRC kinase inhibitors with 266 imatinib on intracellular calcium levels, autophagy, and apoptosis in CML-PBL cells. Cell Cycle 12(17).

267 Guo C, Hou X, Dong L, Dagostino E, Greasley S, Ferre R, Marakovits J, Johnson MC, Matthews D, 268 Mroczkowski B, Parge H, Vanarsdale T, Popoff I, Piraino J, Margosiak S, Thomson J, Los G, Murray 269 BW. 2009. Structure-based design of novel human Pin1 inhibitors (I). Bioorg Med Chem Lett 270 19(19):5613-6.

271 Johnson DE, Redner RL. 2015. An ATRActive future for differentiation therapy in AML. Blood Rev $272 \quad 29(4): 263-8$.

273 Liao X-H, Zhang AL, Zheng M, Li M-Q, Chen CP, Xu H, Chu Q-S, Yang D, Lu W, Tsai T-F, Liu H, 
274 Zhou XZ, Lu KP. 2017. Chemical or genetic Pin1 inhibition exerts potent anticancer activity against 275 hepatocellular carcinoma by blocking multiple cancer-driving pathways. Sci Rep 7:43639.

276 Liao Y, Wei Y, Zhou X, Yang JY, Dai C, Chen YJ, Agarwal NK, Sarbassov D, Shi D, Yu D, Hung MC. 277 2009. Peptidyl-prolyl cis/trans isomerase Pin1 is critical for the regulation of PKB/Akt stability and 278 activation phosphorylation. Oncogene 28(26):2436-2445.

279 Liou Y-C, Ryo A, Huang H-K, Lu P-J, Bronson R, Fujimori F, Uchida T, Hunter T, Lu KP. 2002. Loss of 280 Pin1 function in the mouse causes phenotypes resembling cyclin D1-null phenotypes. Proc Natl Acad Sci 281 U S A 99(3):1335-40.

282 Lu KP, Finn G, Lee TH, Nicholson LK. 2007. Prolyl cis-trans isomerization as a molecular timer. Nat 283 Chem Biol 3(10):619-629.

284 Lu KP, Hanes SD, Hunter T. 1996. A human peptidyl-prolyl isomerase essential for regulation of mitosis. 285 Nature 380(6574):544-547.

286 Lucchetti C, Caligiuri I, Toffoli G, Giordano A, Rizzolio F. 2013. The Prolyl Isomerase Pin1 Acts 287 Synergistically with CDK2 to Regulate the Basal Activity of Estrogen Receptor $\alpha$ in Breast Cancer. PLoS 288 One 8(2):e55355.

289 Montagna R La, Caligiuri I, Giordano A, Rizzolio F. 2013. Pin1 and nuclear receptors: A new language? 290 J Cell Physiol 228(9).

291 Montagna R La, Caligiuri I, Maranta P, Lucchetti C, Esposito L, Paggi MG, Toffoli G, Rizzolio F, 292 Giordano A. 2012. Androgen receptor serine 81 mediates Pin1 interaction and activity. Cell Cycle 293 11(18):3415-20.

294 Moore JD, Potter A. 2013. Pin1 inhibitors: Pitfalls, progress and cellular pharmacology. Bioorg Med 295 Chem Lett 23(15):4283-4291. 
296 Notario B, Zamora M, Viñas O, Mampel T. 2003. All-trans-retinoic acid binds to and inhibits adenine 297 nucleotide translocase and induces mitochondrial permeability transition. Mol Pharmacol 63(1):224-31.

298 Ochoa WF, Torrecillas A, Fita I, Verdaguer N, Corbalán-García S, Gomez-Fernandez JC. 2003. Retinoic 299 Acid Binds to the C2-Domain of Protein Kinase C $\alpha \dagger$. Biochemistry 42(29):8774-8779.

300 Poli G, Giuntini N, Martinelli A, Tuccinardi T. 2015. Application of a FLAP-consensus docking mixed 301 strategy for the identification of new fatty acid amide hydrolase inhibitors. J Chem Inf Model 55(3).

302 Poli G, Lapillo M, Granchi C, Caciolla J, Mouawad N, Caligiuri I, Rizzolio F, Langer T, Minutolo F, 303 Tuccinardi T. 2018. Binding investigation and preliminary optimisation of the 3-amino-1,2,4-triazin$3045(2 \mathrm{H})$-one core for the development of new Fyn inhibitors. J Enzyme Inhib Med Chem 33(1).

305 Poli G, Martinelli A, Tuccinardi T. 2016. Reliability analysis and optimization of the consensus docking 306 approach for the development of virtual screening studies. J Enzyme Inhib Med Chem 31.

307 Regazzi MB, Iacona I, Gervasutti C, Lazzarino M, Toma S. 1997. Clinical pharmacokinetics of tretinoin. 308 Clin Pharmacokinet 32(5):382-402.

309 Rizzolio F, Caligiuri I, Lucchetti C, Fratamico R, Tomei V, Gallo G, Agelan A, Ferrari G, Toffoli G, 310 Klein-Szanto AJ, Giordano A. 2013. Dissecting Pin1 and phospho-pRb regulation. J Cell Physiol $311228(1): 73-7$.

312 Rizzolio F, Lucchetti C, Caligiuri I, Marchesi I, Caputo M, Klein-Szanto AJ, Bagella L, Castronovo M, 313 Giordano A. 2012. Retinoblastoma tumor-suppressor protein phosphorylation and inactivation depend on 314 direct interaction with Pin1. Cell Death Differ 19(7).

315 Roberti A, Rizzolio F, Lucchetti C, Leval L De, Giordano A. 2011. Ubiquitin-mediated protein 316 degradation and methylation-induced gene silencing cooperate in the inactivation of the INK4/ARF locus 317 in Burkitt's lymphoma cell lines. Cell Cycle 10(1). 
318 Russo Spena C, Stefano L De, Palazzolo S, Salis B, Granchi C, Minutolo F, Tuccinardi T, Fratamico R, 319 Crotti S, D’Aronco S, Agostini M, Corona G, Caligiuri I, Canzonieri V, Rizzolio F. 2018. Liposomal 320 delivery of a Pin1 inhibitor complexed with cyclodextrins as new therapy for high-grade serous ovarian 321 cancer. J Control Release 281:1-10.

322 Ryo A, Nakamura M, Wulf G, Liou YC, Lu KP. 2001. Pin1 regulates turnover and subcellular 323 localization of beta-catenin by inhibiting its interaction with APC. Nat Cell Biol 3(9):793-801.

324 Schenk T, Stengel S, Zelent A. 2014. Unlocking the potential of retinoic acid in anticancer therapy. Br J 325 Cancer 111(11):2039-2045.

326 Subedi A, Shimizu T, Ryo A, Sanada E, Watanabe N, Osada H. 2016. Discovery of novel selenium 327 derivatives as Pin1 inhibitors by high-throughput screening. Biochem Biophys Res Commun 474(3):528328533.

329 Suizu F, Ryo A, Wulf G, Lim J, Lu KP. 2006. Pin1 Regulates Centrosome Duplication, and Its 330 Overexpression Induces Centrosome Amplification, Chromosome Instability, and Oncogenesis. Mol Cell 331 Biol 26(4):1463-1479.

332 Tuccinardi T, Granchi C, Rizzolio F, Caligiuri I, Battistello V, Toffoli G, Minutolo F, Macchia M, 333 Martinelli A. 2014a. Identification and characterization of a new reversible MAGL inhibitor. Bioorg Med 334 Chem 22(13):3285-3291.

335 Tuccinardi T, Poli G, Dell'Agnello M, Granchi C, Minutolo F, Martinelli A. 2015. Receptor-based virtual 336 screening evaluation for the identification of estrogen receptor $\beta$ ligands. J Enzyme Inhib Med Chem 337 30(4):662-670.

338 Tuccinardi T, Poli G, Romboli V, Giordano A, Martinelli A. 2014b. Extensive consensus docking 339 evaluation for ligand pose prediction and virtual screening studies. J Chem Inf Model 54(10). 
340 Wei S, Kozono S, Kats L, Nechama M, Li W, Guarnerio J, Luo M, You M-H, Yao Y, Kondo A, Hu H, 341 Bozkurt G, Moerke NJ, Cao S, Reschke M, Chen C-H, Rego EM, Lo-Coco F, Cantley LC, Lee TH, Wu 342 H, Zhang Y, Pandolfi PP, Zhou XZ, Lu KP. 2015. Active Pin1 is a key target of all-trans retinoic acid in 343 acute promyelocytic leukemia and breast cancer. Nat Med 21(5):457-466.

344 Zhao H, Cui G, Jin J, Chen X, Xu B. 2016. Synthesis and Pin1 inhibitory activity of thiazole derivatives. 345 Bioorg Med Chem 24(22):5911-5920. 


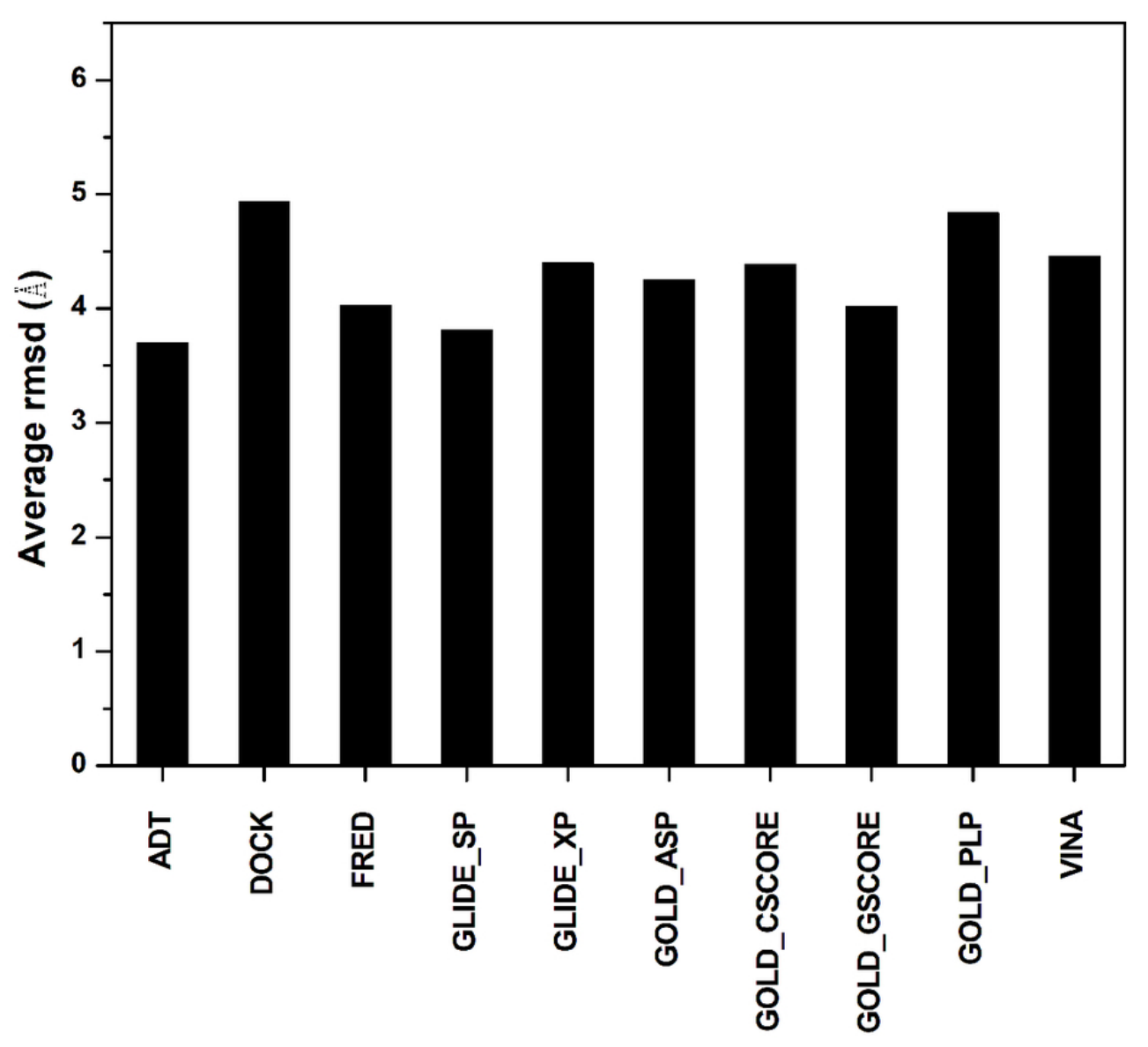

Figure. 1. Average root-mean-square deviations (RMSDs) for ten cross-docking procedures. $80 \times 72 \mathrm{~mm}(300 \times 300 \mathrm{DPI})$ 


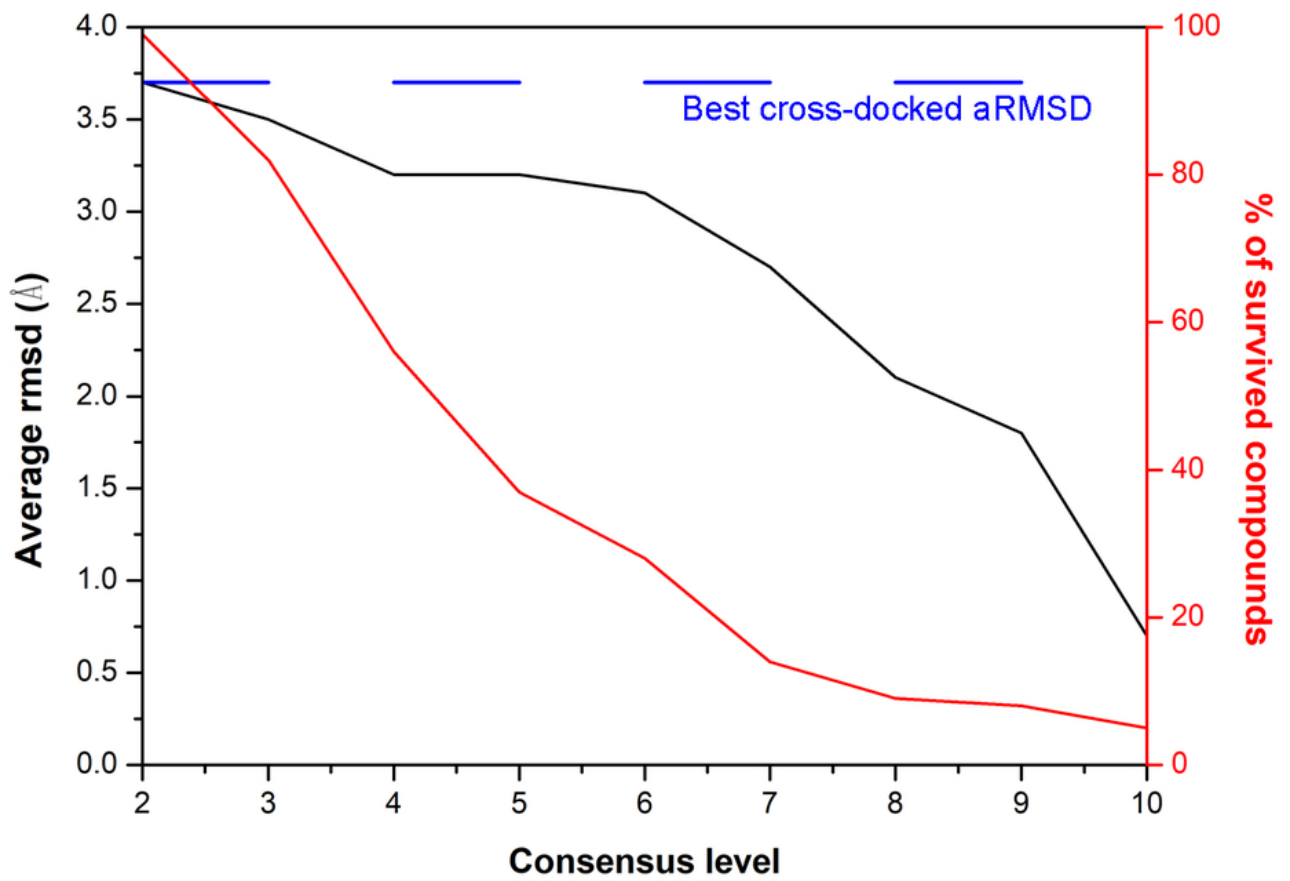

Figure 2. Results of consensus docking. Black line, average RSMD of the consensus docking; red line, percentage of survived compounds; blue interrupted line, best average RMSD obtained with the single docking program AutoDock.

$80 \times 55 \mathrm{~mm}(300 \times 300$ DPI $)$ 


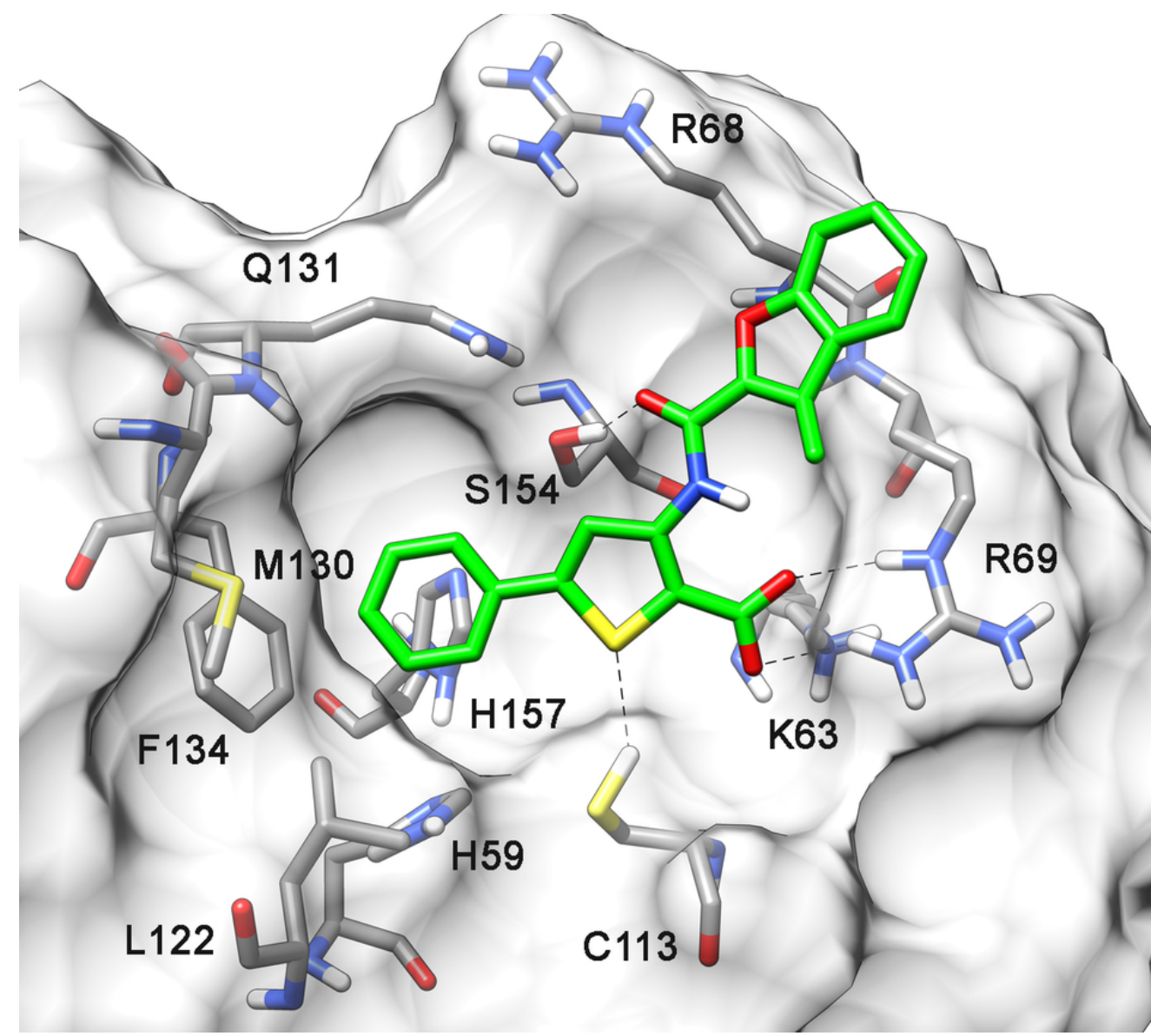

Figure 3. Putative binding pose of VS10 (green) in the binding site of PIN1. The most relevant ligandprotein interactions are marked.

$80 \times 72 \mathrm{~mm}(300 \times 300$ DPI $)$ 


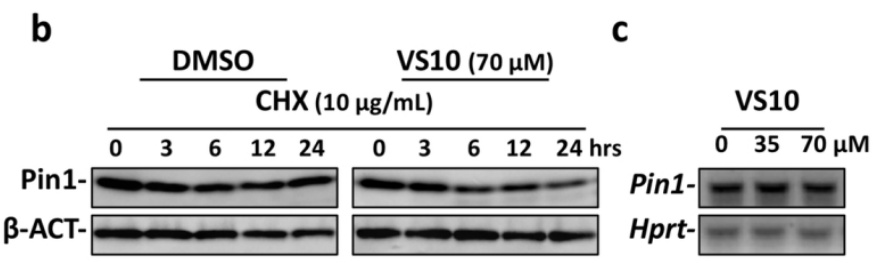

Fig. 4. Compound VS10 targets PIN1 to the proteasome. (a) NIH3T3 fibroblasts were treated with $35 \mu M$ and $70 \mu \mathrm{M}$ VS10 for $48 \mathrm{~h}$, followed by $10 \mu \mathrm{M}$ proteasomal inhibitor MG132 or vehicle for $6 \mathrm{~h}$. (b) Fibroblasts were treated with $70 \mu \mathrm{M}$ VS10 or vehicle for $24 \mathrm{~h}$ followed by $10 \mu \mathrm{g} / \mathrm{mL}$ cycloheximide ( $\mathrm{CHX}$ ) for the indicated times. (c) Cells were treated as in (a), and PIN1 mRNA was amplified by PCR.

$$
139 \times 31 \mathrm{~mm}(300 \times 300 \mathrm{DPI})
$$



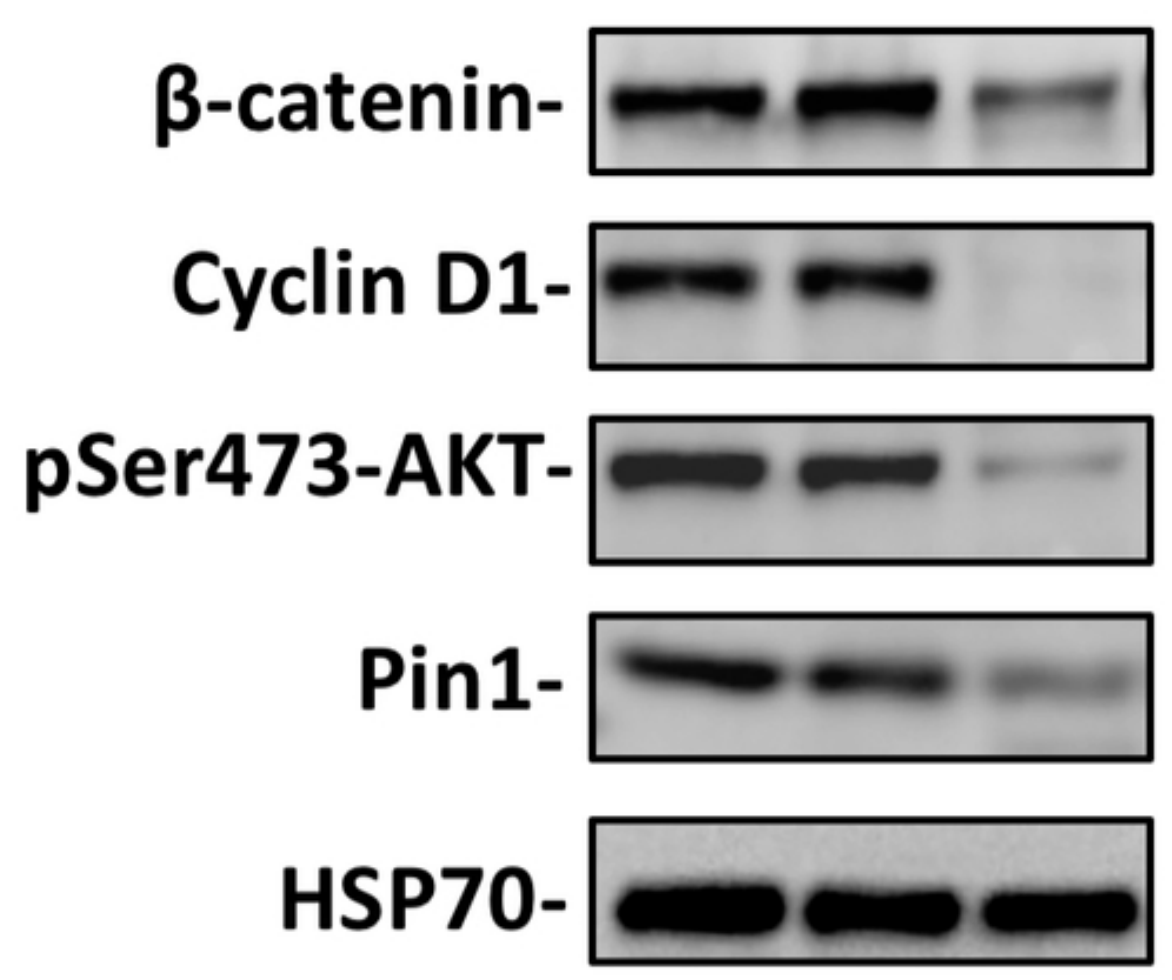

Figure 5. Compound VS10 has inhibitory effects on PIN1 target proteins. OVCAR3 cells were treated with 10 $\mu \mathrm{M}$ ATRA (control) or $70 \mu \mathrm{M}$ VS10, then lysed and subjected to western blotting. HSP70 was used as a control for sample loading.

$43 \times 56 \mathrm{~mm}(300 \times 300 \mathrm{DPI})$ 


(2)




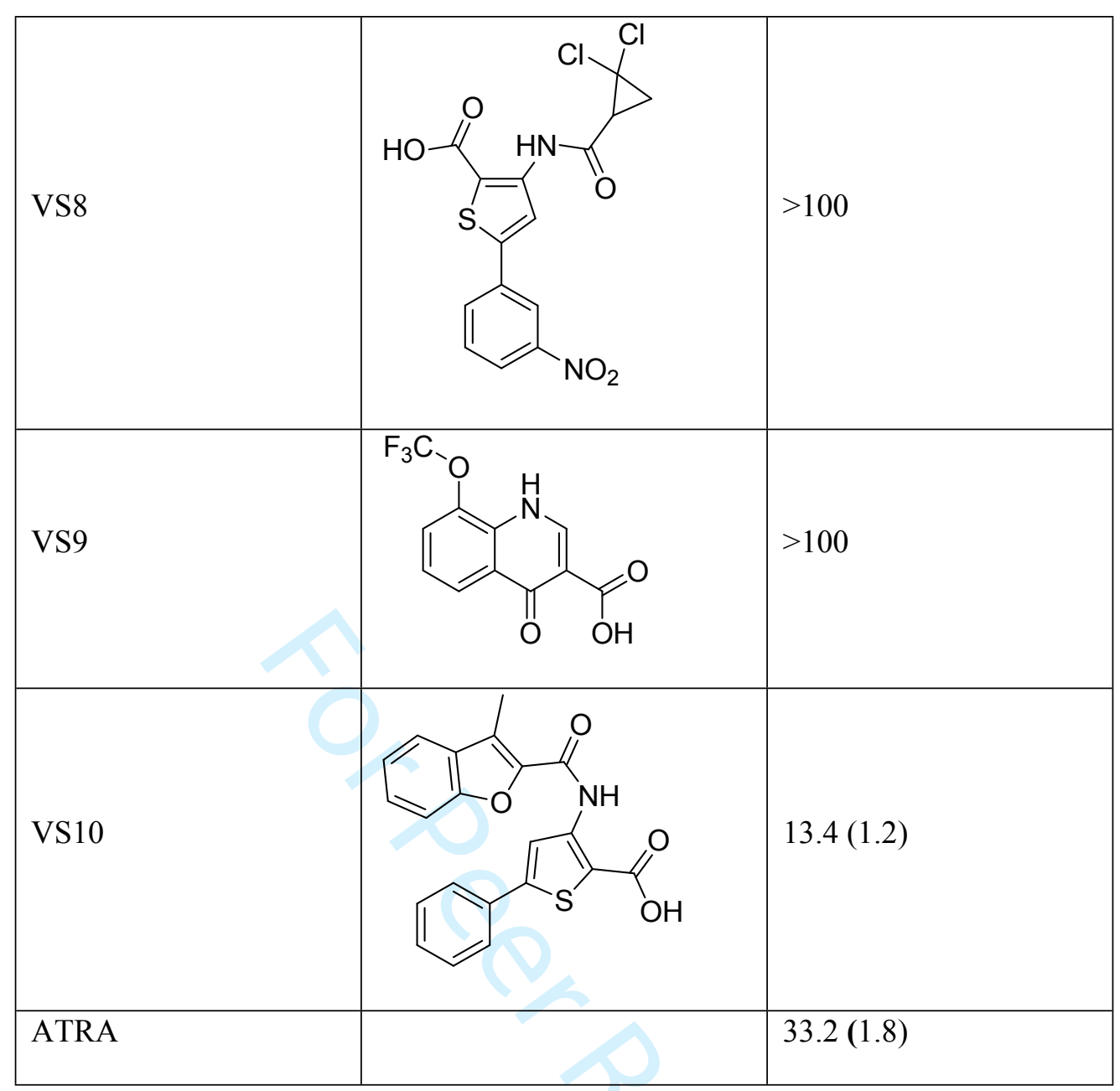

Table 1. Structures and half-maximal inhibitory concentrations $\left(\mathrm{IC}_{50}\right)$ on human PIN1 isomerization (SensoLyte Green assay), for ten compounds selected by virtual screening and for ATRA (positive control). Values are mean (SD). 


\begin{tabular}{ll}
\hline Cell line & $\mathbf{I C}_{\mathbf{5 0}}(\boldsymbol{\mu M})$ \\
\hline OVCAR3 & $53.9(26.0)$ \\
OVCAR5 & $75.0(25.7)$ \\
SKOV3 & $76.4(14.5)$ \\
A2780 & $53.9(21.5)$
\end{tabular}

Table 2. $\mathrm{IC}_{50}$ of VS10 in ovarian cancer cell lines. Values are mean (SD). 